\title{
Eichrechtliche Aspekte beim Smart Metering
}

Dr. Helmut Többen/PTB

\section{Kurzfassung}

In diesem Beitrag wird das Smart Metering aus eichrechtlicher und metrologischer Sicht behandelt. Das Gesamtsystem der Metrologie in Deutschland wird beschrieben, die wesentlichen Aspekte des Eichrechts insbesondere im Hinblick auf das Smart Metering werden ausführlicher behandelt und der mit der Verabschiedung des neuen Energiewirtschaftsgesetzes aktuell diskutierte Ansatz wird vorgestellt.

\section{Einleitung}

Mit der Verabschiedung des neuen Energiewirtschaftsgesetzes am 28. Juli 2011 soll die Einführung des Smart Meterings in Deutschland vorangetrieben werden. Das Smart Metering soll zum Einen den Haushaltskunden ermuntern, seine Verbrauchsmengen (Strom, Gas, Wärme, Wasser) zu reduzieren und zum Anderen auf der Versorgungsseite ein besseres Steuern der Bedarfsströme ermöglichen. Hierzu sollen insbesondere aktuelle/momentane Verbrauchswerte beim Verbraucher angezeigt und vom Versorger/Lieferanten in kurzen Zeitabständen abgerufen werden. Mittels variabler Tarife, dies können lastvariable, zeitvariable oder sich kurzfristig ändernde Tarife sein, soll zudem eine gezielte, monetär spürbare Steuerung des Verbraucherverhaltens erreicht werden. Sobald Messwerte nicht nur für Informationszwecke verwendet werden, sondern auch für Abrechnungszwecke, sind nach wie vor metrologische und eichrechtliche Aspekte zu berücksichtigen.

Am weitesten vorgeschritten ist das Thema Smart Metering im Bereich der Elektrizität. Die Steuerung der Stromnetze und das sehr zeitnahe Reagieren auf Fluktuationen, die von Schwankungen im Verbraucherverhalten (Haushalt, Industrie, Elektrofahrzeuge) oder bei der Einspeisung (Windenergie-, Photovoltaikanlagen) herrühren können, stellen eine besondere Herausforderung dar. In diesem Zusammenhang ist vor allem auch das Thema Smart Grid zu nennen, das als Synonym für optimiert arbeitende Netze steht. Bei der Smart Grid Technologie werden die von den Smart Metern gelieferten Messwerte als Eingangsgrößen benötigt.

Die Steuerung von Gasnetzen, die ein hohes Potenzial an Speicherkapazität besitzen, ist hingegen weniger zeitkritisch. Neueste Entwicklungen verfolgen aber ein stärkeres Zusammenwirken beider Netze. So ist beabsichtigt, aus überschüssiger Windenergie synthetisches Erdgas zu erzeugen („Windgas“), in das Erdgasnetz einzuspeisen und bspw. anderorts in Gaskraftwerken zur Stromerzeugung zu verbrauchen. Der Vollständigkeit halber sei noch erwähnt, dass die Sparten Wasser und Wärme aktuell nicht im Fokus der Diskussionen um das Thema Smart Metering stehen.

In diesem Beitrag wird nur ein Teilbereich des Smart Meterings betrachtet. Er konzentriert sich auf die metrologischen und eichrechtlichen Aspekte, mit dem Versuch, dem Leser in aller Kürze die Grundzüge der Metrologie und des Eichrechts sowie die Anwendung des Eichrechts auf technische Lösungen zu vermitteln. 


\section{Metrologie}

Metrologie ist die Wissenschaft und Anwendung des richtigen Messens. Richtiges Messen bedeutet, dass die eingesetzte Messtechnik und die angewendeten Verfahren korrekte und zuverlässige Ergebnisse liefern und dass das Vertrauen in die ermittelten Messwerte quantifiziert wird. In der Metrologie kommt Messtechnik zum Einsatz, die im Vorfeld durch eine Technik mit geringerer Messunsicherheit geprüft oder kalibriert worden ist. Letztendlich werden in einem stufigen Prozess alle Messwerte über die nationalen Normale auf das SI-Einheitensystem zurückgeführt.

Die Angabe von Messunsicherheitswerten, die den Messwerten beigeordnet werden, liefert eine Aussage über das Vertrauen in die Messrichtigkeit des Messverfahrens. Auf metrologisch höchstem Niveau kommen die sogenannten nationalen Normale zum Einsatz, die ein Messen mit höchstmöglicher Genauigkeit und Präzision erlauben. Auf diesem messtechnischen Niveau werden auch die kleinstmöglichen Messunsicherheitswerte erreicht.

Die Physikalisch-Technische Bundesanstalt (PTB) steht als nationales Metrologieinstitut dem Gesamtsystem der Metrologie in Deutschland vor. Sie ist als technische Oberbehörde im Bundesministerium für Wirtschaft und Technologie (BMWi) angesiedelt und realisiert, z. T. mit Partnerinstitutionen, die bestmögliche Messtechnik/die bestmöglichen Messverfahren für die messtechnische Erfassung einer Vielzahl von physikalischen Größen. In Zusammenarbeit mit akkreditierten Kalibrierlaboratorien sowie den Eichbehörden und den staatlich anerkannten Prüfstellen wird gewährleistet, dass vor Ort bspw. bei der Fertigung industrieller Produkte, bei der Messung im medizinischen Bereich oder beim Erwerb von Verbrauchsgütern die angewendeten Messverfahren ebenfalls korrekte und zuverlässige Ergebnisse liefern.

\section{Messung und Abrechnung von Verbrauchsgütern}

Die Messung und Abrechnung von Verbrauchsgütern wie Elektrizität, Gas, Wasser und Wärme unterliegt dem gesetzlichen Messwesen und damit dem Eichrecht. Der Schutz des Verbrauchers, der sich auf die erhaltenen Messergebnisse verlassen möchte, wird durch die Bestimmungen und die Anwendung des Eichgesetzes sichergestellt. Außerdem sorgt das Eichrecht auch für einen lauteren Wettbewerb. Im Kern verlangt das Eichrecht die Messrichtigkeit und Messbeständigkeit der eingesetzten Geräte sowie den Schutz der Geräte vor Manipulation. Zudem verlangt das Eichrecht, dass die in Rechnung gestellten Verbrauchswerte auf Messwerten basieren und vom Verbraucher überprüfbar und nachvollziehbar darzustellen sind.

Vor der Liberalisierung der Energiemärkte waren für Haushaltskunden die Abläufe bzgl. der Messung und Abrechnung von Verbrauchsgütern noch recht überschaubar und einfach. Der Verbraucher hat seine benötigten Ressourcen direkt vom Versorger bezogen und auch direkt mit ihm abgerechnet. Der zu zahlende Rechnungsbetrag orientierte sich an den vertraglich vereinbarten Konditionen (bspw. Arbeits- und Grundpreis) und den einmal im Jahr am geeichten Haushaltszähler ablesbaren und damit auch überprüfbaren Messwerten. Mit Umsetzung der Liberalisierung und insbesondere mit Einführung des Smart Meterings wurden Veränderungen angestoßen, die zwar neue Möglichkeiten bieten, die z. T. aber auch neue technische und organisatorische Lösungen erfordern. 
Wie eingangs schon erwähnt, verfolgt das Smart Metering ein zeitnahes Informieren des Verbrauchers über seine Verbrauchsmengen und ein verbessertes Steuern der Versorgungsnetze. Mit der Einführung neuer Tarifstrukturen sollen zudem zusätzliche monetäre Anreize zum Energiesparen geschaffen und damit ein Glätten von Lastspitzen in den Netzen erreicht werden. Des weiteren ist mit der Einführung des Smart Meterings eine Erweiterung des Funktionsumfanges angedacht, wie bspw. die Einführung des Pre-Payments oder das Betätigen von Absperreinrichtungen aus der Ferne. Technische Voraussetzungen für das Smart Metering sind der Aufbau einer bidirektionalen Kommunikationsinfrastruktur für einen Datentransfer, bspw. über offene Netze, sowie das Ersetzen der klassischen Verbrauchszähler durch Zähler mit der Möglichkeit zur Kommunikation. All diese Neuerungen müssen mit den metrologischen und eichrechtlichen Aspekten sowie den Datensicherheits- und Datenschutzaspekten in Einklang gebracht werden, siehe hierzu auch letztes Kapitel.

\section{Verfahren und Akteure im Eichrecht}

Bis zur Umsetzung der MID (Measurement Instrument Directive) im Jahre 2006 erfolgte in Deutschland das Inverkehrbringen der Verbrauchsmessgeräte für Elektrizität, Gas, Wasser und Wärme nach dem Eichgesetz [1] und der Eichordnung [2]. Nach Erteilung einer durch die PTB ausgesprochene Bauartzulassung und einer anschließenden Ersteichung durch eine Eichbehörde oder eine staatlich anerkannte Prüfstelle durften die Geräte beim Nutzer/Endkunden installiert und in Betrieb genommen werden. Nach Ablauf der Eichgültigkeitsdauer wurden durch Stichprobenverfahren oder durch eine Nacheichung die Messrichtigkeit und Messbeständigkeit der Geräte für weitere Jahre sichergestellt.

Mit Einführung der MID [3] liegt das europaweite Inverkehrbringen von Verbrauchsmessgeräten in einem erhöhten Maß in der Verantwortlichkeit der Gerätehersteller. Für das Inverkehrbringen müssen sie eine Konformitätserklärung ihrer Geräte vorlegen können, die bspw. durch eine erfolgreiche Konformitätsbewertung eines Gerätebaumusters (Modul B) in Kombination mit einer erfolgreichen Bewertung des Qualitätsmanagementsystems des Herstellers (Modul D) erteilt werden kann. Die Bewertung nach den unterschiedlichen Modulen wird jeweils durch eine benannte Stelle für MID-Funktionen durchgeführt. Je nach Benennung können dies private Stellen, die Eichbehörden oder die PTB sein. Nach diesem Ansatz kann ein Gerät mit entsprechender Konformitätsbewertung dann in ganz Europa in den Verkehr gebracht werden.

Nach dem Inverkehrbringen der Geräte soll im Rahmen einer Marktaufsicht ein gemeinsames Schutzniveau auf EU-Ebene sichergestellt werden. Die Durchführung der Marktaufsicht unterliegt dem nationalen Recht und wird in Deutschland durch die Eichbehörden ausgeübt. Durch proaktive und reaktive Maßnahmen wird die erklärte Konformität der Geräte noch vor der Inbetriebnahme überprüft. Ebenfalls dem nationalen Recht unterliegt die Überwachung der korrekten Verwendung der Messgeräte während ihres Betriebes. Hierzu gehört die Nacheichung bspw. zur Verlängerung der Eichgültigkeit und die Befundprüfung, wenn bspw. ein Verbraucher die Messrichtigkeit seines Haushaltszählers anzweifelt. Beides kann von staatlich anerkannten Prüfstellen und den Eichbehörden durchgeführt werden. Die Eichbehörden sind zudem für die Verwenderüberwachung zuständig. Hierfür werden Zwischenkontrollen angesetzt. 


\section{Eichrechtliche materielle Anforderungen}

An das Gerät und an die Verwendung der Messwerte werden seitens des Eichrechts materielle Anforderungen gestellt. Das Gerät muss richtig und beständig messen sowie vor Manipulationen geschützt sein bzw. Manipulationsversuche müssen am Gerät erkennbar sein. Außerdem verlangen die MID und die Eichordnung, dass alle abrechnungsrelevanten Werte auf einer dem Verbraucher ohne Hilfsmittel zugänglichen und der metrologischen Kontrolle unterliegenden Sichtanzeige dargestellt werden.

Reine IT-Lösungen wie das im Rahmen des BMWi-Projektes Vernet entwickelte SELMA-Konzept [4] erlauben eine Messwerterfassung per Fernauslesung und wären in Kombination mit einer geeichten Sichtanzeige beim Verbraucher auch MIDkonform. Das SELMA-Konzept wurde in Zusammenarbeit mit Geräteherstellern, den Eichbehörden und der PTB entwickelt und ist bereits Basis für einige in Deutschland zugelassene Messsysteme. Auch für Smart Metering bietet dieses Konzept eine geeignete technische Lösung insbesondere in Verbindung mit einer Einheit, die beim Verbraucher ein spartenübergreifendes Sammeln und Weiterleiten von Verbrauchsdaten ermöglicht. Mittels kryptografischer Signierung, die die Integrität (Unverfälschtheit) und Authentizität (Zuordnung zum Messort) der Messwerte sicherstellt, können die Messwerte über offene Netze bspw. in die Zentrale des Lieferanten zu Abrechnungszwecken, an den Netzbetreiber zu Steuerungszwecken und an den Verbraucher zur Darstellung der Verbrauchswerte übertragen werden. Aus Gründen der Vertraulichkeit/des Datenschutzes (nicht Gegenstand des Eichrechts) muss neben einer Signierung auch eine Verschlüsselung der Daten gefordert werden - auch hierzu der Hinweis auf das letzte Kapitel.

In diesem Zusammenhang sollte auch erwähnt werden, dass die technische Ausstattung für das Weiterleiten bzw. Duplizieren bereits geeicht gemessener und beim Verbraucher über eine geeichte Sichtanzeige darstellbarer Messwerte nicht der Eichpflicht unterliegt. Die PTB-A50.7 [5] fordert allerdings, dass die Datenübertragung zur Vermeidung von Fehlern mit bestimmten Mindeststandards durchzuführen ist. Die Anzeige und die Verwendung von Messwerten oder Messfunktionen für den eigenen Bedarf (interne Steuerung von Betriebsabläufen, Überwachung und Steuerung des eigenen Verbrauchs usw.) unterliegen ebenfalls nicht der Eichpflicht.

Ein wichtiger eichrechtlicher Aspekt ist die Rückverfolgbarkeit der Messwerte. Demnach darf eine Rechnung nur auf Messwerten beruhen, die mit eichrechtlich kontrollierten Messgeräten bzw. Messsystemen ermittelt worden sind. Wenn Messwerte ein Messgerät - in dem sie noch gegen Verfälschungen geschützt sind verlassen und die Verbindung zwischen der Messwerterfassung und der Sichtanzeige über eine manipulationsunsichere Kommunikationsleitung (offene Netze) erfolgt, müssen sie mittels kryptografischer Verfahren vor Manipulation geschützt werden. Sie dürfen während ihrer gesamten Lebenszeit, insbesondere bis zum Ablauf der Einspruchsfrist des Kunden, nicht verändert werden. Auf jeden Fall muss der Kunde eine evt. stattgefundene Veränderung erkennen können und bis zum Ablauf der Einspruchsfrist auch die Möglichkeit haben, die der Rechnung zugrunde gelegten Werte mit den Originalwerten zu vergleichen. Deshalb sind die abrechnungsrelevanten Werte in einer geeichten Einheit zu speichern. 
Die konventionelle technische Lösung ist die Speicherung am Messort in einem eichrechtlich kontrollierten Messgerät oder einer entsprechenden Zusatzeinrichtung. Zur Rechnungsüberprüfung muss der Kunde die Möglichkeit erhalten, auf die gespeicherten Messwerte zuzugreifen und sie mit eichrechtlich kontrollierten, zugelassenen bzw. konformitätsbewerteten Hilfsmitteln sichtbar zu machen und auf Unverfälschtheit zu überprüfen. Zum Zwecke der Überprüfung müssen alle abrechnungsrelevanten Werte auch aus der Rechnung hervorgehen. Bei Mehrtarifanwendungen muss auch eine eindeutige Zuordnung der Messwerte zu den jeweilig geltenden Tarifen stattfinden. Auf diese Weise kann nicht nur der Kunde seine Rechnung überprüfen, sondern der Verwender/Rechnungssteller kann auch seinen Zahlungsanspruch nachweisen.

\section{Tarifstrukturen}

Grundsätzlich enthält das Eichrecht keine Regelungen für Tarife. Wie bereits erläutert, stellt das Eichrecht sicher, dass richtig gemessen wird und dass die richtigen Messwerte den betroffenen Seiten, insbesondere dem Kunden, zur Prüfung der Richtigkeit seiner Rechnung zur Verfügung stehen. Ein regelkonformes Messgerät trennt hard- oder softwaremäßig sauber zwischen Kommunikations- und Messfunktionen. Ein Update der Kommunikationssoftware und der Software, die für die Ausführung der nicht eichrechtlichen Funktionen notwendig ist, ist dann einfach und ohne eichrechtliche Einschränkungen möglich. Wenn lediglich ein Tarif zwischen den Vertragspartnern besteht, wird der Preis zeitunabhängig ermittelt. Seit mehr als hundert Jahren sind aber auch zeitabhängige, über Schaltuhren aktivierte Tarife bekannt. Für Haushaltskunden wurden bisher hauptsächlich zeitvariable Tarife für Hoch- und Niedertarifregelungen (HT/NT) angewendet. Zur Messwerterfassung werden hierzu Zweitarifzähler eingesetzt.

Es gibt auch die Möglichkeit, ferngesteuert Tarife zu aktivieren oder Zeiträume mit bestimmten Tarifen vertraglich zu regeln. In diesen Fällen muss der Zähler innerhalb der den Tarifen zugeordneten Zeiten die jeweiligen Verbrauchswerte messen und anzeigen. Mittels sogenannter Mehrtarifzähler werden abhängig vom aktiven Tarif die akkumulierten Verbrauchswerte in den einzelnen Zeiträumen aufsummiert und angezeigt. Gleichzeitig muss dem Verbraucher aber auch der aktuell gültige Tarif am Gerät kenntlich gemacht werden.

Bei Lastgangzählern und zeitgesteuerten Tarifen muss dem Verbraucher zudem die Zeit angezeigt werden. Solche Lösungen sind zugelassen, werden vielfach eingesetzt und stellen seit Jahren den Stand der Technik im Bereich der Sondervertragskunden (Jahres-Stromverbrauch > 100.000 kWh oder JahresGasverbrauch $>1.500 .000 \mathrm{kWh}$ ) dar. So wird die registrierende Lastgangmessung (RLM) millionenfach im Elektrizitäts- und hunderttausendfach im Gasbereich eingesetzt. Die Verbrauchswerte werden viertelstündlich (Strom) bzw. stündlich (Gas) erfasst und angezeigt, im Gerät gespeichert und über ein angeschlossenes Kommunikationsmodul zu Bilanzierungszwecken mehrmals täglich abgefragt.

\section{Rechtliche Vorgaben - zeitlicher Rückblick}

Auf europäischer Ebene wurde im Jahr 2006 die Richtlinie für Energieeffizienz und Energiedienstleistungen (EDL-RL) 2006/32/EG verabschiedet mit dem Ziel, dass die Verbraucher durch Informationen über ihr Verbrauchsverhalten Anregungen zum 
Energiesparen erhalten. National wurde dies umgesetzt durch die Änderung des Energiewirtschaftsgesetzes (EnWG), durch das Gesetz zur Öffnung des Messwesens bei Strom und Gas für mehr Wettbewerb (8. April 2008) und durch die Messzugangsverordnung (17. Oktober 2008). Hiernach sollten ab dem 1. Januar 2010 konventionelle Zähler mit Einverständnis der Nutzer gegen kommunikative Zähler ausgetauscht werden bzw. wenn die technische Machbarkeit und wirtschaftliche Zumutbarkeit gegeben ist, in Neubauten und bei größeren Renovierungsarbeiten verbaut werden.

Im Jahr 2009 hat die Bundesnetzagentur (BNetzA) von der Bundesregierung den Auftrag erhalten, eine Gutachterstellungnahme zu erarbeiten, die Aussagen liefert zu den technischen, wirtschaftlichen und rechtlichen Aspekten eines flächendeckenden Einsatzes fernauslesbarer Zähler, zu den Aspekten der Belastungsregistierung bzw. Lastgangmessung und zu den Möglichkeiten und Voraussetzungen lastvariabler Tarife. Im gleichen Jahr hat die EU Kommission an CEN, CENELEC und ETSI ein Mandat (M/441) zur Normung von Zusatzfunktionen ausgesprochen mit dem Ziel, genormte Schnittstellen und Austauschformate für eine sichere bidirektionale Kommunikation sowie eine flexible Architektur zur Unterstützung einfachster und komplexer Anwendungen und zur Anpassung aktueller und zukünftiger Kommunikationsmedien zu entwickeln.

Obwohl schon recht früh von verschiedenen Stromanbietern Zähler mit Smart Meter Funktionalitäten angeboten wurden, herrschte in den Jahren 2009/2010 wegen fehlender Investitionssicherheit doch eher eine abwartende Haltung am Markt vor. Bspw. wurden MID zugelassene Multi-Tarifzähler in Kombination mit einem rückwirkungsfreien Kommunikationsmodul angeboten, die abgesehen vom Eigenverbrauchsanteil als technische Lösung den Grundgedanken des Smart Meterings und gleichzeitig die Anforderungen des Eichrechts erfüllten bzw. auch heute noch erfüllen.

Die BNetzA hat im März 2010 ihre Gutachterstellungnahme der Bundesregierung vorgelegt [6]. In der Stellungnahme, die vorwiegend die Stromseite betrachtet, wurde eine getrennte Bestandsaufnahme und Bewertung hinsichtlich moderner Messsysteme und variabler Tarife durchgeführt. Da der letzte Entwurfsstand des Energieeffizienzgesetzes zur Umsetzung der EDL-Richtlinie mit Vorschlag zur Änderung von §21b EnWG von der Bundesregierung bisher nicht verabschiedet wurde, wurde in der Stellungnahme noch keine Gesamt-System-Lösung vorgegeben. Nach dem dritten Richtlinienpaket (RL2009/72/EG und RL2009/73/EG) kann noch bis zum 3. September 2012 eine Wirtschaftlichkeitsanalyse durchgeführt werden, die dann als Basis für weitere Überlegungen und Festlegungen seitens der BNetzA dienen soll. Die BNetzA hat jedoch in ihrem Bericht ein für weitere Entwicklungen offenes modulares Gerätekonzept empfohlen. Dieses Konzept beinhaltet einen Basiszähler (vgl. EDL21-Zähler) mit vorgegebenen Funktionalitäten und Schnittstellen, der letztendlich zu einem Smart Meter ausgebaut werden könnte. Zur Einführung einer Bilanzierung bei Privatkunden/Haushaltskunden in Verbindung mit variablen Tarifen wurde von der BNetzA eine Zählerstandsgangerfassung empfohlen, die mit im Markt befindlichen Geräten bedient werden kann. 


\section{Rechtliche Vorgaben - aktueller Stand}

Da auf europäischer Ebene die Arbeiten zum Normungsmandat M/441 schleppend vorangehen und in den Gesetzestexten die Aspekte zum Datenschutz und zur Informationssicherheit bisher wenig Berücksichtigung fanden, hat die Bundesregierung mit der Überarbeitung des neuen Energiewirtschaftsgesetzes [7] u.a. ein nationales Vorantreiben zur erfolgreichen Einführung des Smart Meterings verfolgt. Das am 28. Juli 2011 verabschiedete EnWG schreibt jetzt den Einbau sogenannter Messsysteme ab dem 1. Januar 2013 vor. Gemäß §21 EnWG sind Messsysteme in Kommunikationsnetze eingebundene Messeinrichtungen zur Erfassung der elektrischen Energie, die gleichzeitig den Anschlussknoten für weitere/andere MID-Verbrauchs- und Einspeisemessgeräte darstellen. Sie sollen zudem zur Datenerhebung, -verarbeitung, -speicherung, -überprüfung und insbesondere zur gesicherten Datenübermittlung eingesetzt werden. Hierbei sind die eichrechtlichen Vorschriften sowie die Anforderungen an Datenschutz, Datensicherheit und Interoperabilität einzuhalten.

Das zentrale neue Element innerhalb des Messsystems ist das sogenannte Smart Meter Gateway (SM-GW) - ein bidirektional arbeitendes Kommunikationsmodul mit erweitertem Funktionsumfang. Gemäß der vom Bundesamt für Sicherheit in der Informationstechnik (BSI) noch in der Entwicklung befindlichen Technischen Richtlinie TR-03109 [8] soll mit dem SM-GW eine kryptografisch gesicherte Kommunikation erreicht werden, um die Authentizität, die Integrität und die Vertraulichkeit von Daten in den öffentlichen Netzen zu gewährleisten. Um Einfallstore für Manipulationsversuche zu minimieren, fordert die TR ebenso eine gesicherte Kommunikation zwischen dem SM-GW und den einzelnen, anzuschließenden Verbrauchsmessgeräten. Das SM-GW soll als kommunikative Schnittstelle außerdem die technische Voraussetzung für Homeautomation-Prozesse und für die Visualisierung von Informationen allgemeiner und verbraucherspezifischer Art (Verbrauchswerte, Tarifinformationen, Vergleichsdaten) darstellen. Die Einhaltung der Anforderungen an Datensicherheit und Datenschutz sowie an die Interoperabilität werden im Rahmen eines Zertifizierungsprozesses festgestellt.

Außerdem kann das SM-GW auch eichrechtlich betrachtet werden müssen. Werden von MID-Zählern stammende Messwerte im SM-GW mit einer Zeit- oder Ereignisstempelung versehen, die für die abrechnungsrelevante Zuordnung zu unterschiedlichen Tarifen genutzt wird, unterliegt das SM-GW dem Eichrecht. Vergleichbar mit der eichrechtlichen Betrachtung der Mehrtarifzähler und Lastgangzähler sind dann insbesondere auch die eichrechtlichen Kriterien hinsichtlich der Rückverfolgbarkeit der Rechnungswerte und der Darstellung der Messwerte beim Verbraucher vor Ort zu gewährleisten.

Insgesamt wurde mit der Verabschiedung des neuen EnWG ein spannender Prozess angeschoben, der zurzeit (Anfang 2012) an verschiedenen Stellen noch Klärungsund Diskussionsbedarf beinhaltet. Münden soll dieser Prozess in einer noch zu formulierenden Rechtsverordnung, die zusammen mit der fertig gestellten TR-03109 die im EnWG aufgestellten Anforderungen in konkrete Vorgaben umsetzt. Auf dem Weg dorthin erfährt das BMWi nicht nur Unterstützung durch das BSI sondern auch durch die PTB, die BNetzA und den BfDI. Außerdem werden regelmäßig die Verbände und Interessenvertreter im Rahmen von Workshops und Konsultationsverfahren in den Entwicklungsprozess eingebunden. Das Ziel unter den 
gegebenen Randbedingungen ist die Findung wirtschaftlich sinnvoller und technisch praktikabler Lösungen, die die Einführung des Smart Meterings erleichtern.

\section{Schlussfolgerungen}

Neben der Sicherstellung eines lauteren Wettbewerbs steht beim Eichrecht der Verbraucherschutz im Vordergrund. So verlangt das Eichrecht eine korrekte und zuverlässige messtechnische Erfassung der Verbrauchswerte sowie die Nachvollziehbarkeit der Abrechnung durch die Möglichkeit des Überprüfens aller abrechnungsrelevanter Werte durch den Verbraucher - auch bei Anwendung variabler Tarife. Eichrechtlich betrachtet könnten mit den heute vorliegenden technischen Gerätelösungen in Kombination mit einem Kommunikationsmodul bereits Smart Meter konformitätsbewertet bzw. zugelassen werden. Inwiefern diese Geräte „smart" sind, ist eine andere Frage. Mit der Verabschiedung des neuen Energiewirtschaftgesetzes soll die Einführung des Smart Meterings in Deutschland einen Vortrieb erhalten. Zur Gewährleistung hoher Sicherheitsstandards in der Versorgungsinfrastruktur sind hierbei insbesondere die Anforderungen an Datenschutz und Datensicherheit zu berücksichtigen. Das neue technische Gerät ist hierbei das Smart Meter Gateway, das als zentrale Schnittstelle dient, eine gesicherte Kommunikation gewährleistet und eichrechtlich relevante Funktionen ausführen kann.

\section{Literatur}

[1] Gesetz über das Meß- und Eichwesen (Eichgesetz).

http://www.gesetze-im-internet.de/bundesrecht/eichg/gesamt.pdf

[2] Eichordnung.

http://www.gesetze-im-internet.de/mwg-internal/de5fs23hu73ds/progress?id=PiAPqM3jJ8

[3] Richtlinie 2004/22/EG des Europäischen Parlaments und des Rates vom 31. März 2004 über Messgeräte.

http://eur-lex.europa.eu/LexUriServ/site/de/oj/2004/I_135/I_13520040430de00010080.pdf

[4] Norbert Zisky (Hrsg.): Das SELMA-Projekt - Konzepte, Modelle, Verfahren. PTB-Bericht PTB-IT12, Braunschweig und Berlin, März 2005.

[5] Anforderungen an elektronische und softwaregesteuerte Messgeräte und Zusatzeinrichtungen für Elektrizität, Gas, Wasser und Wärme (PTB-A 50.7).

http://www.ptb.de/de/org/q/q3/q31/ptb-a/ptb-a.htm\#sec_50

[6] Wettbewerbliche Entwicklungen und Handlungsoptionen im Bereich Zähl- und Messwesen und bei variablen Tarifen.

http://www.bundesnetzagentur.de/cae/servlet/contentblob/151968/publicationFile/6321/BerichtZaehIM esswesenpdf.pdf

[7] Gesetz über die Elektrizitäts- und Gasversorgung (Energiewirtschaftsgesetz). http://www.gesetze-im-internet.de/bundesrecht/enwg_2005/gesamt.pdf

[8] BSI TR-03109 Anforderungen an die Interoperabilität der Kommunikationseinheit eines intelligenten Messsystems für Stoff und Energiemengen

https://www.bsi.bund.de/ContentBSI/Publikationen/TechnischeRichtlinien/tr03109/index_htm.html 